Methods Rituximab-treated patients from the Karolinska University Hospital $(n=107)$ were enrolled. LON was defined as an absolute neutrophil count $<1,500$ cells/ $\mu \mathrm{L}$, occurring four weeks to two years following treatment, or later in cases of sustained B cell depletion, provided that other apparent causes were excluded. B cell activating factor (BAFF), a proliferationinducing ligand (APRIL), IL-6, granulocyte-macrophage colonystimulating factor (GM-CSF), and granulocyte colony-stimulating factor (G-CSF) were measured using ELISA prior to and post-treatment.

Results Thirty-two patients (29.9\%) developed LON after a median time of 201.5 days (IQR: 66.8-322.0). Thirteen patients were admitted to the hospital; ten developed fever, and three developed critical conditions. BAFF levels increased from baseline (median: $0.62 \mathrm{ng} / \mathrm{mL}$; IQR: 0.42-1.07) through the post-treatment measurement, both in patients who developed LON (median: $1.73 \mathrm{ng} / \mathrm{mL}$; IQR: 1.03-2.56; $\mathrm{p}=0.005$ ) and patients who did not (median: 1.03 ; IQR: $0.65-1.55$; $\mathrm{p}=0.001$ ), with significantly higher BAFF levels in the LON group $(p=0.021)$. APRIL levels were higher in the LON group both at baseline (median: 1.54 versus $1.15 \mathrm{ng} / \mathrm{mL} ; \mathrm{p}=0.027$ ) and post-treatment (median: 2.39 versus $1.11 \mathrm{ng} / \mathrm{mL} ; \mathrm{p}=0.011)$. IL-6 and GM-CSF levels decreased in the non-LON group $(\mathrm{p}<0.001)$. Cumulative rituximab and cyclophosphamide doses were found to be associated with the development of agranulocytosis $(p=0.022$ and $p=0.021$, respectively).

Conclusion Post-rituximab LON is a common complication in SLE. Although the phenomenon was self-limiting in most cases, a few patients developed life-threatening conditions; this highlights the importance of regular surveillance for neutrophil counts, fever and infections. Distinct roles of BAFF and APRIL are implicated; BAFF might contribute to granulopoiesis disruptions, whereas APRIL might have a value in distinguishing predisposed patients.

\section{PS7:135 AMG 592 IS AN INVESTIGATIONAL IL-2 MUTEIN THAT INDUCES HIGHLY SELECTIVE EXPANSION OF REGULATORY T CELLS}

${ }^{1} \mathrm{~N}$ Tchao, ${ }^{2} \mathrm{KS}$ Gorski, ${ }^{3} \mathrm{~T}$ Yuraszeck, ${ }^{4} \mathrm{SJ}$ Sohn, ${ }^{5} \mathrm{~K}$ Ishida, ${ }^{6} \mathrm{H}$ Wong, ${ }^{7} \mathrm{~K}$ Park. ${ }^{7}$ Amgen Inc., Department of Early Development, South San Francisco, USA; ${ }^{2}$ Amgen Inc., Department of Clinical Biomarkers, South San Francisco, USA; ${ }^{3}$ Amgen Inc., Department of Pharmacokinetics and Drug Metabolism, Thousand Oaks, USA; ${ }^{4}$ Amgen Inc., Department of Inflammation Research, South San Francisco, USA; ${ }^{5}$ Amgen Inc., Department of Comparative Biology and Safety Sciences, Thousand Oaks, USA; ${ }^{6}$ Amgen Inc., Department of Clinical Pharmacology, South San Francisco, USA; ${ }^{7}$ Amgen Inc., Department of Global Safety, Cambridge, USA

\subsection{6/lupus-2018-abstract. 178}

Background AMG 592 is an investigational IL-2 mutein designed for greater regulatory T-cell (Treg) selectivity and longer half-life than recombinant IL-2 (aldesleukin). We investigated the tolerability of AMG 592 and its effects on expansion of Tregs, conventional effector T-cells (Tcon), and natural killer (NK) cells.

Methods AMG 592 activity, in comparison with aldesleukin, was assessed by in vitro phosphorylated STAT5 (pSTAT5), Treg/Tcon/NK cell expansion, and cytokine production in primary human peripheral blood mononuclear cells (hPBMC). Effects on body temperature (Temp), C-reactive protein (CRP), and peripheral Treg/Tcon/NK cell numbers were evaluated in cynomolgus monkeys (CM). In an FIH study, healthy volunteers received single ascending SC AMG 592 doses $(n=6 /$ dose;
8 cohorts) or placebo $(n=2 /$ dose $)$ for 28 days. Adverse events (AEs), pharmacokinetics (PK), pharmacodynamics, and cytokines were evaluated.

Results In hPBMC cultures, AMG 592 caused more selective Treg response (pSTAT5, proliferation) and lower proinflammatory cytokine levels than aldesleukin. Dose-dependent expansion of FoxP3 + Tregs was associated with increased Temp and CRP in aldesleukin-treated but not in AMG 592-treated CM. In the FIH study, AMG 592 was well tolerated, with no serious AEs. The most common AE across cohorts was grade 1 painless erythema at/near the injection site which resolved without treatment. Preliminary PK results indicate dose-related increases in AMG 592 serum exposure. AMG 592 caused robust, dose-dependent Treg expansion relative to Tcon in all treated individuals. Expanded Tregs had increased CD25 and FoxP3, and were enriched for recent thymic emigrants. At the highest dose, increase in Treg: Tcon ratio peaked at day 8 ( $\sim 4$ fold vs baseline) and remained elevated up to day 29. AMG 592-mediated Treg expansion was highly selective, with no directional change in NK cell numbers and minimal increase in Tcon; there were no increases in serum proinflammatory cytokines IL-6, TNF $\alpha$, or IFN- $\gamma$ above the limits of detection.

Conclusion AMG 592 caused dose-dependent, selective Treg expansion in healthy volunteers. Lack of proinflammatory cytokines and reduced inflammation markers suggest a wider therapeutic margin, and sustained Treg elevation implies less frequent dosing, compared with aldesleukin. Further investigation of AMG 592-induced Treg-mediated restoration of immune homeostasis in inflammatory and autoimmune diseases is warranted.

\section{PS7:136 APOPTOTIC EFFECT OF BLYS ON ENDOTHELIAL CELLS AND ENDOTHELIAL PROGENITOR CELLS IS MEDIATED BY BLYS RECEPTORS AND IS REVERTED BY BELIMUMAB}

FR Spinelli, C Barbati, F Ceccarelli, T Colasanti, L Massaro, F Morello, C Garufi, C Alessandri, G Valesini, F Conti. Sapienza Università di Roma - Dipartimento di Medicina Interna e Specialità Mediche, Reumatologia, Rome, Italy

\subsection{6/lupus-2018-abstract.179}

Circulating endothelial progenitor cells (EPCs) are markers of endothelial function; their reduction and functional impairment in patients with Systemic Lupus Erythematosus (SLE), partially account for endothelial dysfunction. In murine models of atherosclerosis, treatment with a B Lymphocyte Stimulator (BLyS) reduced atherosclerotic plaque size and progression.

In a case study on SLE 20 women, our group confirmed a decrease in the number of EPCs, with a significant increase after treatment with Belimumab (BLM).

The aims of this study were: to evaluate the ex vivo and in vitro effects of BLyS stimulation and inhibition on the EPC colonies and on endothelial cells; to investigate BLyS receptor expression of on EPCs and endothelial cells.

EPCs were isolated from peripheral blood mononuclear cells and defined as CD34+/VEGF-R2 + double positive cells. To evaluate the ability to form colonies, the EPCs of 2 SLE patients and 2 healthy controls were cultured on fibronectincoated dishes and incubated with BlyS or Blys and BLM and counted after 7 days.

Apoptosis of EPCs and endothelial cell line (EA.hy926) was evaluated after 6, 12 and 24 hours incubation with BLyS and after 6 hour with BLyS and BLM. EPC and EA.hy926 were 
also incubated with anti-B activating factor-receptor (BAFF-R) antibodies, B-cell maturation antigen (BCMA) and transmembrane activator and calcium modulator and cyclophilin ligand (CAML) interactor (TACI), then analysed by cytofluorimetry.

The number of EPC colonies in patients was lower than in controls; moreover, colonies were poorly organised compared to controls; BLM incubation restored the structure of the colonies. After 6 hours of incubation, BLyS $(20 \mathrm{ng} / \mathrm{ml})$ induced apoptosis of EPC and EA.hy926; co-incubation with BLM inhibited the apoptotic effect. Both EPCs and EA.hy926 expressed BAFF-R (MFI=3.8 and 1.5 respectively) and BCMA $(\mathrm{MFI}=1.25$ and 1.15$)$; EPCs also express TACI $(\mathrm{MFI}=1.4)$.

The results of this study showed:

1. a quantitative and qualitative alteration of colonies in patients, restored after ex vivo and in vitro BLM treatment;

2. the apoptotic effect of BLyS on EPC and endothelial cells inhibit by BLM and

3. the preferential expression of BAFF-R on the surface of EPC and EA.hy926.

\section{PS7:137 THE USE OF BELIMUMAB IN RECALCITRANT CUTANEOUS LUPUS: A CASE REPORT}

${ }^{1} \mathrm{M}$ Gilio, ${ }^{1} \mathrm{G}$ Tramontano, ${ }^{1} \mathrm{MS}$ Cutro, ${ }^{1} \mathrm{~T}$ Carbone, ${ }^{1} \mathrm{~V}$ Picerno, ${ }^{2} \mathrm{U}$ Bottoni, ${ }^{1} \mathrm{~A}$ Padula, 'S D'Angelo. 'Rheumatology Institute of Lucania (IRel), the Rheumatology Department of Lucania, San Carlo Hospital of Potenza, Potenza, Italy; ${ }^{2}$ Department of Health Sciences, University of Catanzaro Magna Graecia, Catanzaro, Italy

\subsection{6/lupus-2018-abstract.180}

Background The anti-BAFF monoclonal antibody, belimumab, was approved about five years ago by the US Food and Drug Administration for the treatment of adult SLE patients. The utility of belimumab for management of resistant systemic lupus erythematosus (SLE) has been demostrated but concerning skin manifestations only scarce evidences have been reported. We describe our experience of using this new drug for the successful management of recalcitrant cutaneous lupus. Case report A 38-year-old man with a five year history of SLE presented, in May 2017, at our outpatient clinic for a disease flare with severe cutaneous involvement. On examination the patient presented malar rash and erythematous-infiltrated discoid lesions in the region of head and neck and erythematosus papules also on the extensor surface of the hands. Additional tests showed also systemic involvement by detecting low levels of C3 and C4, leukopenia (WBC 3000/ $\mathrm{LL}$ ) and positivity of ANA (1:1280 by IFI) and anti-dsDNA $(42.8 \mathrm{UI} / \mathrm{ml}$ by ELISA, nv $<30 \mathrm{UI} / \mathrm{ml}$ ). SLE Disease Activity Index (SLEDAI) was 9, Cutaneous Lupus Disease Area and Severity index- activity and damage scores (CLASI) was 22 for activity and 1 for damage and Physician Global Assessment (PGA) was $8 \mathrm{~cm}$. The patient failed previous treatment with HCQ, MTX, AZA, MMF and at time of our observation was taking, since December 2016, prednisone (12,5 mg daily) without improvement. Belimumab was added to concomitant steroid therapy at recommended dose $(10 \mathrm{mg} / \mathrm{kg})$. Early as 3 months after its initiation Belimumab therapy led to impressive clinical improvement in the lesions upper the hands and slighter in that in the region of head. Belimumab use also provided a significant steroid-sparing effect as well as facilitating the rapid improvement in skin symptoms and in systemic involvement.

Conclusion In this case report, the addition of belimumab to steroid monotherapy, in patient who failed previous

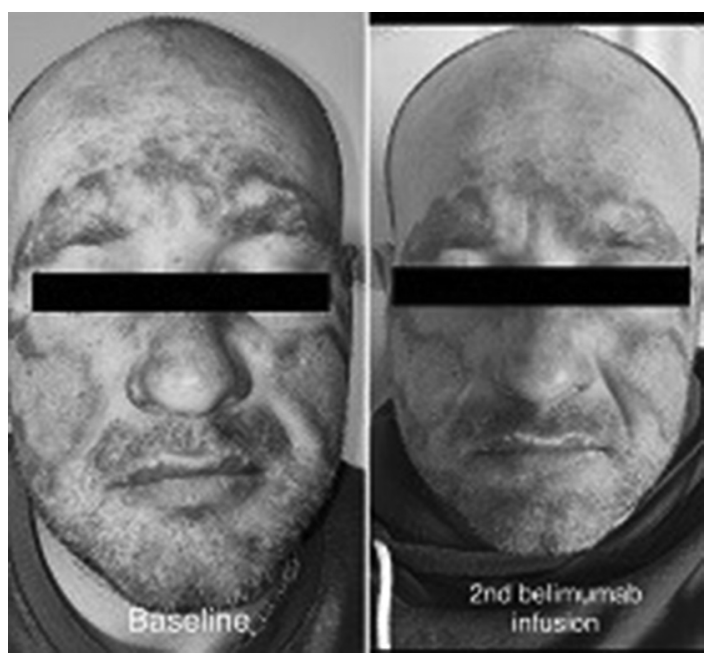

Abstract PS7:137 Figure 1

immunosuppressive treatment improved the signs and symptoms of refractory cutaneous lupus. This report highlights the utility of belimumab for the treatment of severe skin involvement in SLE refractory to conventional therapies. Additional studies should be performed to assess the use of belimumab in the treatment of cutaneous lupus.

\section{PS7:138 NEW STRATEGY THERAPY FOR LUPUS NEPHRITIS WITH PERSISTENT PROTEINURIA}

S Algergawy. Benha University Faculty of Medicine Rheumatology and Rehabilitation Department, Beha, Egypt

\subsection{6/lupus-2018-abstract.181}

Background Glomerulonephritis and renal failure represent one of the most life-threatening manifestations of systemic lupus erythematosus. Many patients show persistent proteinuria despite conventional therapy (anti-inflammatory and immunosuppressive therapies). $f$. Vitamin $D$ is immune modulator thought to be a potent inhibitor of the RAAS (renin-anigotensin-aldosterone system) which increase in kidney damage. Vitamin D deficiency is common in systemic lupus erythematosus. So Correcting vitamin D deficiency may play important role for treatment lupus nephritis

Aim The aim of This study will detect $t$ the potential role of high supplementation of vitamin D therapy as anti-proteinuric effects in the treatment of lupus nephritis on conventional therapy with persistent proteinuria.

Patients and methods Ninty patients with with lupus nephritis and persistent proteinuria despite conventional therapy will be recruited. They will be treated with vitamin D and follow up for 24 months. Proteinuria, renal function, lupus disease activity, serum and urinary inflammatory markers and urinary angiostatin will be monitored. the mean vitamin $\mathrm{D}$ in the patient group was $10.7+7.9 \mathrm{ng} / \mathrm{ml}$. vitamin $\mathrm{D}$ supplementation depend on severity of deficient and weight of patient s. twenty five patients with lupus nephritis without vitamin D supplementation as control group.

Results Our results show that reduction in protinuria as measured by urinary protien creatinine (UP/C) ratio in 24 hour collection at $12(\mathrm{r}, 0.61 . \mathrm{p}<0.001)$, and 24 weeks (r, 0.65. $\mathrm{p}<0.001$ ), compared with base line, all patients completed all 